\title{
Optimality and duality in set-valued optimization using higher-order radial derivatives
}

\author{
Guolin Yu*, Xiangyu Kong \\ Institute of Applied Mathematics, Beifang University of Nationalities
}

(Received: 9 October 2015; Accepted: 26 April 2016)

\begin{abstract}
This paper is devoted to the study of optimality conditions and duality theory for a set-valued optimization problem. By using the higher-order radial derivative of a set-valued map, we establish Fritz John and Kuhn-Tucker types of necessary and sufficient optimality conditions for a weak minimizer of a set-valued optimization problem under the assumption that set-valued maps in the formulation of objective and constraint maps are near cone-subconvexlike. As an application of the optimality conditions, we prove weak, strong and converse duality theorems for Mond-Weir and Wolfe types dual problems.
\end{abstract}

Keywords Radial derivative, Optimality conditions, Set-valued optimization,Weak minimizer, Duality

AMS 2010 subject classifications Primary 90C29, 90C46; Secondary 26B25.

DOI: $10.19139 /$ soic.v4i2.175

\section{Introduction}

In recent years, there has been an increasing interest in the investigation of higher-order (generalized) derivatives, higher-order optimality conditions and duality theory for set-valued optimization problems. Several kinds of higher-order derivatives have been developed for set-valued mappings by different authors. To some extent, higherorder derivatives in the related literatures can be divided into two categories: First, the existences of higherorder derivatives depend on the choice of lower-order directions, for instance, higher-oder contingent (adjacent) derivatives [1], the generalized higher-order contingent (adjacent) derivatives [2], cone-directed higher-order contingent (adjacent) derivatives [3], higher-order generalized contingent (adjacent) epiderivatives [4], higher-order weak epiderivatives [5], and variational sets $[6,7,8]$, etc.; Second, the direction of higher-order derivatives does not depend on lower-order direction, for example, Higher-order Studniarski derivative $[9,10,11]$ enjoys this advantage. In fact, much attention has been paid upon optimality conditions and related topics for vector optimization by using Studniarski derivatives [9, 10,11, 12, 13]. Another meaningful concept in this direction is radial derivative [14]. Radial derivatives take some advantages of other kinds of derivatives, and are proved to be applicable to nonconvex problems and global optimal solutions [14, 15, 16, 17]. Recently, Anh etc. [18] made a higher-order extensions for radial derivatives and presented optimality conditions in type of separating of sets for several kinds of efficiency in set-valued optimization. However, the classical forms, such as: Fritz John and Kuhn-Tucker types, have not been proposed. This is a motivation for our present work. For more recent works related to higher-order radial derivatives, we refer the readers to $[19,20]$.

On the other hand, convex analysis is a powerful tool for the investigation of optimal solutions of set-valued optimization problems. Various notions of generalized convexity have been introduced to weaken convexity. One

\footnotetext{
*Correspondence to: Guolin Yu (Email: guolin_ yu@126.com). Institute of Applied Mathematics, Beifang University of Nationalities, Yinchuan, Ningxia 750021, P. R. China.
}

ISSN 2310-5070 (online) ISSN 2311-004X (print)

Copyright (C) 2016 International Academic Press 
of such generalizations in set-valued analysis is called cone-convexity [21], which plays a very important role in setvalued optimization. Based upon this concept, some scholars developed further generalizations of cone-convexity to vector optimization involving set-valued maps. For example, cone-preinvexity [22], cone-convexlikeness [23], cone-subconvexlikeness [24], generalized cone-subconvexlikeness [25] and near cone-subconvexlikeness [25] etc. Among these notions, the nearly cone-subconvexlikeness is more general than other generalized convexities. In this paper, we shall take use of nearly cone-subconvexlikeness as the weaker condition on convexity assumption.

Duality assertions allow to study a minimization problem through a maximization problem and to know what one can expect in the best case. Duality has many applications in optimization, and it has provided many unifying conceptual insights into economics and management science. So, it is not surprising that duality is one of the important topics in set-valued optimization. There are several papers dedicated to duality theory of set-valued optimization by using higher-order derivatives [1, 2, 3, 4, 5]. Among results obtained in this field, Mond-Weir type dual problems have been received much attention. In this note, we present a Wolfe tpype dual problem and prove its duality theorems.

Based upon the above observations, this paper is focused on higher-order radial derivatives of set-valued maps and weak minimizer of a set-valued optimization problem under weaker condition on convexity. The purpose of this paper is two folds: first, we establish the optimality conditions for weak minimizers in Fritz John and KuhnTucker types; second, we provide an employment of optimality conditions for weak minimizers to obtain some duality results for higher-order Mond-Weir and Wolfe types dual problems. The layout of this paper is as follows: Section 2 contains some well-known definitions and results used in the paper. In Section 3 and Section 4, we give the optimality conditions and duality theorems of weakly minimizers, respectively.

\section{Preliminaries}

Throughout the paper, it is assumed that $X, Y$ and $Z$ are real normed linear spaces with topological dual $X^{*}, Y^{*}$ and $Z^{*}$, repectively. For any $x \in X$ and $x^{*} \in X^{*}$, the canonical form between $X$ and $X^{*}$ is denoted by $x^{* T} x$. We assume $A$ is a nonempty subset of $Y$, the closure of $A$ is denoted by $\operatorname{cl} A$ and the cone generated by $A$ is denoted by cone $(A)=\left\{\lambda a: a \in A, \lambda \in \mathbb{R}_{+}\right\}$, where $\mathbb{R}_{+}$denotes the set of nonnegative real numbers. Let $D \subset Y$ and $E \subset Z$ be pointed closed convex cones, which are supposed to be solid, i.e., int $D \neq \emptyset$ and $\operatorname{int} E \neq \emptyset$. We define

$$
D^{*}=\left\{y^{*}: y^{* T} d \geq 0, \forall d \in D\right\},
$$

and similarly for $E^{*}$.

Let $F: X \rightarrow 2^{Y}$ be a set-valued mapping. The set

$$
\operatorname{dom}(F):=\{x \in X: F(x) \neq \emptyset\},
$$

is called the domain of $F$. The set

$$
\operatorname{graph}(F):=\{(x, y) \in X \times Y: y \in F(x)\}
$$

is called the graph of the map $F$. For a subset $M \subset X$, we follow the convention $F(M)=\bigcup_{x \in M} F(x)$.

Let $m \geq 1$ be an integer. Anh etc. [18] proposed the following higher-order radial derivatives:

Definition 2.1 (see [18]) Let $F: X \rightarrow 2^{Y}$ be a set-valued map and $u \in X$. The $m$ th-order outer radial derivative of $F$ at $(\bar{x}, \bar{y}) \in \operatorname{graph}(F)$ is defined as

$$
\begin{aligned}
D_{R}^{m} F(\bar{x}, \bar{y})(u):= & \left\{v \in Y: \exists t_{n}>0, u_{n} \in X, v_{n} \in Y \text { for } \forall n\right. \\
& \text { such that } \left.\left(u_{n}, v_{n}\right) \rightarrow(u, v), n \rightarrow+\infty \text { and } \bar{y}+t_{n}^{m} v_{n} \in F\left(\bar{x}+t_{n} u_{n}\right)\right\} .
\end{aligned}
$$

Lemma 2.1 (see [18]) Let $(\bar{x}, \bar{y}) \in \operatorname{graph}(F)$. Then, for all $x \in X$ and $m \geq 1$,

$$
F(x)-\bar{y} \subset D_{R}^{m} F(\bar{x}, \bar{y})(x-\bar{x}) .
$$


Remark 2.1 It is worthy mentioned that this higher-order radial derivatives does not need any convexity assumptions. However, not all of derivatives possess this property. For example: the limit set of a set-valued mapping (see Definition 1 in [26]) enjoys this property under additional pseudoconvexity condition.

The generalized convexity used in this paper is called nearly cone-subconvexlikeness.

Definition 2.2 (see [25]) Let $M \subset X$ be a nonempty set and the set-valued mapping $F: M \rightarrow 2^{Y}$ is said to be near $D$-subconvexlike on $M$ if $\operatorname{cl}[\operatorname{cone}(F(M)+D)]$ is convex.

Remark 2.2 The notion of nearly cone-subconvexlikeness includes various known generalizations of convexity given in the literatures as special cases, for example, cone-preinvexity [22], cone-convexlikeness [23], conesubconvexlikeness [23] and generalized cone-subconvexlikeness [24], etc.

The following conclusion is the alternative theorem for near $D$-subconvexlike set-valued map, which is necessary for the results in the next section.

Lemma 2.2 (see [25]) Let $M \subset X$ be a nonempty set and $F: M \rightarrow 2^{Y}$. Suppose that $F$ is near $D$-subconvexlike map on $M$. Then exactly one of the following conclusions holds:

(i) $\exists x \in M$ such that $F(x) \cap(-\operatorname{int} D) \neq \emptyset$,

(ii) $\exists y^{*} \in D^{*} \backslash\{0\}$ such that $y^{* T} y \geq 0, \forall y \in F(x), \forall x \in M$.

Let $S \subset X$ be a nonempty set, $F: S \rightarrow 2^{Y}$ and $G: S \rightarrow 2^{Z}$ be two set-valued maps and we assume that

$$
\operatorname{dom} F=\operatorname{dom} G=S .
$$

For $x \in X$, we use the notation $(F \times G)(x)$ to denote $(F(x), G(x))$. We consider the following set-valued optimization problem:

$$
\text { (SOP) } \begin{cases}\text { minimize } & F(x) \\ \text { subject to } & G(x) \cap(-E) \neq \emptyset, \\ & x \in S,\end{cases}
$$

The feasible set of problem (SOP) is denoted by $\Omega:=\{x \in S: G(x) \cap(-E) \neq \emptyset\}$.

Definition 2.3 (see [18]) Let $(\bar{x}, \bar{y}) \in \operatorname{graph}(F)$ with $\bar{x} \in S .(\bar{x}, \bar{y})$ is said to be a weak minimizer of problem (SOP) if

$$
(F(S)-\bar{y}) \cap-\operatorname{int} D=\emptyset .
$$

\section{Higher order optimality conditions}

In this section, we establish higher-order necessary and sufficient optimality conditions in Fritz John and KuhnTucker types of weak minimizers to problem (SOP). To begin we present a necessary optimality condition in Fritz John type for a weak minimizer of (SOP).

Theorem 3.1 Let $(\bar{x}, \bar{y}) \in \operatorname{graph}(F)$ and $\bar{z} \in G(\bar{x}) \cap(-E)$. Suppose that $(\bar{x}, \bar{y})$ is a weak minimizer of (SOP) and $(F-\bar{y}) \times G$ is near $D \times E$-subconvexlike. Then, there exists $\left(y^{*}, z^{*}\right) \in\left(D^{*} \times E^{*}\right) \backslash\left\{\left(0_{Y^{*}}, 0_{Z^{*}}\right)\right\}$ such that for all $(y, z) \in D_{R}^{m}(F \times G)(\bar{x}, \bar{y}, \bar{z})(x), x \in \operatorname{dom} D_{R}^{m}(F \times G)(\bar{x}, \bar{y}, \bar{z})$,

$$
y^{* T} y+z^{* T} z \geq 0,
$$

and

$$
z^{* T} \bar{z}=0
$$


Proof Since $(\bar{x}, \bar{y})$ is a weak minimizer of (SOP), we get that

$$
((F \times G)(S)-(\bar{y}, 0)) \cap-\operatorname{int}(D \times E)=\emptyset .
$$

Since $(F-\bar{y}) \times G$ is near $D \times E$-subconvexlike map on $S$, it follows from Lemma 2.2 that there exists $\left(y^{*}, z^{*}\right) \in$ $\left(D^{*} \times E^{*}\right) \backslash\left\{\left(0_{Y^{*}}, 0_{Z^{*}}\right)\right\}$ such that

$$
y^{* T}(y-\bar{y})+z^{* T} z \geq 0, \quad \forall(y, z) \in(F \times G)(S) .
$$

Taking $y=\bar{y}$ and $z=\bar{z}$, we obtain $z^{* T} \bar{z} \geq 0$. Then, it follows from $z^{* T} \bar{z} \leq 0$ (because $\bar{z} \in-E$ ) that $z^{* T} \bar{z}=0$. So,

$$
y^{* T}(y-\bar{y})+z^{* T}(z-\bar{z}) \geq 0, \quad \forall(y, z) \in(F \times G)(S) .
$$

On the other hand, let $(y, z) \in D_{R}^{m}(F \times G)(\bar{x}, \bar{y}, \bar{z})(x)$ for $x \in \operatorname{dom} D_{R}^{m}(F \times G)(\bar{x}, \bar{y}, \bar{z})$, then there exist $\left\{t_{n}\right\}$ with $t_{n}>0$ and $\left\{\left(x_{n}, y_{n}, z_{n}\right)\right\}$ in $X \times Y \times Z$ with $\left(x_{n}, y_{n}, z_{n}\right) \rightarrow(x, y, z)$ such that

$$
(\bar{y}, \bar{z})+t_{n}^{m}\left(y_{n}, z_{n}\right) \in(F \times G)\left(\bar{x}+t_{n} x_{n}\right) .
$$

Thus, $\bar{x}_{n}:=\bar{x}+t_{n} x_{n} \in \operatorname{dom}(F \times G)$ and there are $\left(\bar{y}_{n}, \bar{z}_{n}\right) \in(F \times G)\left(\bar{x}_{n}\right)$ such that

$$
(\bar{y}, \bar{z})+t_{n}^{m}\left(y_{n}, z_{n}\right)=\left(\bar{y}_{n}, \bar{z}_{n}\right) .
$$

Noticing that $\left(y_{n}, z_{n}\right) \rightarrow(y, z)$, we obtain that

$$
\frac{\bar{y}_{n}-\bar{y}}{t_{n}^{m}} \rightarrow y
$$

and

$$
\frac{\bar{z}_{n}-\bar{z}}{t_{n}^{m}} \rightarrow z
$$

So, for large enough $n$, we have

$$
y^{* T}\left(\frac{\bar{y}_{n}-\bar{y}}{t_{n}^{m}}\right)+z^{* T}\left(\frac{\bar{z}_{n}-\bar{z}}{t_{n}^{m}}\right) \geq 0 .
$$

Taking $n \rightarrow+\infty$, we obtain that $y^{* T} y+z^{* T} z \geq 0$. This completes the proof.

Next, we establish a necessary optimality condition in Kuhn-Tucker type. It is derived from Theorem 3.1 by adding the following constraint qualification on the mapping $G$ in (SOP), that is

$$
\operatorname{cl}(\operatorname{cone}(G(S)+E))=Z .
$$

This condition has been used by several authors, for instance: [27, 28]

Theorem 3.2 Let $(\bar{x}, \bar{y}) \in \operatorname{graph}(F), \bar{z} \in G(\bar{x}) \cap(-E)$ and $(F-\bar{y}) \times G$ be near $D \times E$-subconvexlike on $S$. Suppose that $(\bar{x}, \bar{y})$ is a weak minimizer of $(\mathrm{SOP})$ and $\operatorname{cl}(\operatorname{cone}(G(S)+E))=Z$. Then, there exist $y^{*} \backslash\left\{0_{Y^{*}}\right\}$ and $z^{*} \in E^{*}$ such that for all $(y, z) \in D_{R}^{m}(F \times G)(\bar{x}, \bar{y}, \bar{z})(x), x \in \operatorname{dom} D_{R}^{m}(F \times G)(\bar{x}, \bar{y}, \bar{z})$,

$$
y^{* T} y+z^{* T} z \geq 0
$$

and

$$
z^{* T} \bar{z}=0
$$

Proof It follows from Theorem 3.1 that there exists $\left(y^{*}, z^{*}\right) \in\left(D^{*} \times E^{*}\right) \backslash\left\{\left(0_{Y^{*}}, 0_{Z^{*}}\right)\right\}$ such that for all $(y, z) \in$ $D_{R}^{m}(F \times G)(\bar{x}, \bar{y}, \bar{z})(x), x \in \operatorname{dom} D_{R}^{m}(F \times G)(\bar{x}, \bar{y}, \bar{z}), y^{* T} y+z^{* T} z \geq 0$ and $z^{* T} \bar{z}=0$. So, it suffices to prove $y^{*} \neq 0$. From the proof of Theorem 3.1, we get

$$
y^{* T}(y-\bar{y})+z^{* T} z \geq 0, \quad \forall(y, z) \in(F \times G)(S) .
$$


Suppose that $y^{*}=0$. Then $z^{*} \neq 0$ and $z^{* T} z \geq 0$. On the other hand, it is obviously that $z^{* T} e \geq 0$ for all $e \in E$. Noticing that $z^{*}$ is a continuous linear functional, we get

$$
z^{* T} z^{\prime} \geq 0, \forall z^{\prime} \in \operatorname{cl}(\operatorname{cone}(G(S)+E))=Z .
$$

Thus $z^{*}=0$, which is impossible, because $\left(y^{*}, z^{*}\right) \neq\left(0_{Y^{*}}, 0_{Z^{*}}\right)$.

To end this section, we present the following sufficient optimality conditions for a weak minimizer of (SOP). This result and Theorem 3.2 will be applied to the proofs of duality theorems in next section.

Theorem 3.3 Let $(\bar{x}, \bar{y}) \in \operatorname{graph}(F)$ and $\bar{z} \in G(\bar{x}) \cap(-E)$. Suppose that there exist $m \geq 1, y^{*} \in D^{*} \backslash\{0\}$ and $z^{*} \in E^{*}$ such that

$$
y^{* T} y+z^{* T} z \geq 0,(y, z) \in D_{R}^{m}(F \times G)(\bar{x}, \bar{y}, \bar{z})(x), x \in \operatorname{dom} D_{R}^{m}(F \times G)(\bar{x}, \bar{y}, \bar{z}),
$$

and

$$
z^{* T} \bar{z}=0
$$

Then, $(\bar{x}, \bar{y})$ is a weak minimizer of (SOP).

Proof Suppose that $(\bar{x}, \bar{y})$ is not a weak minimizer of (SOP). Then there is $x \in \Omega$ such that

$$
(F(x)-\bar{y}) \cap(-\operatorname{int} D) \neq \emptyset .
$$

Hence, there is $y \in F(x)$ and $z \in G(x) \cap(-E)$ such that

$$
y-\bar{y} \in-\operatorname{int} D .
$$

Noticing that $y^{*} \in D^{*} \backslash\{0\}$, we get that

$$
y^{* T}(y-\bar{y})<0 .
$$

On the other hand, it yields from Lemma 2.1 that

$$
(F \times G)(x)-(\bar{y}, \bar{z}) \subset D_{R}^{m}(F \times G)(\bar{x}, \bar{y}, \bar{z})(x) .
$$

Hence, we get that

$$
y^{* T}(y-\bar{y})+z^{* T}(z-\bar{z}) \geq 0 .
$$

Furthermore, it implies

$$
y^{* T}(y-\bar{y}) \geq-z^{* T}(z-\bar{z})=-z^{* T} z \geq 0,
$$

which is a contradiction.

\section{Duality Theorems}

\subsection{Mond-Weir Type Duality}

In this subsection, for the primal problem (SOP) we consider a higher-order Mond-Weir type dual problem. Let $\left(x^{\prime}, y^{\prime}\right) \in \operatorname{graph}(F)$ and $z^{\prime} \in G\left(x^{\prime}\right) \cap-E$. Considering the following Mond-Weir dual problem (MWD):

(MWD)

$$
\begin{cases}\max & y^{\prime} \\ \text { s.t. } & y^{* T} y+z^{* T} z \geq 0, \forall(y, z) \in D_{R}^{m}(F \times G)\left(x^{\prime}, y^{\prime}, z^{\prime}\right)(x), \\ & \quad \forall x \in \operatorname{dom} D_{R}^{m}(F \times G)\left(x^{\prime}, y^{\prime}, z^{\prime}\right) \\ & z^{* T} z^{\prime} \geq 0 \\ & \left(y^{*}, z^{*}\right) \in\left(D^{*} \backslash\left\{0_{Y^{*}}\right\}\right) \times E^{*}\end{cases}
$$


Denote by $K_{1}$ the set of all feasible points of (MWD), i.e. the set of points $\left(x^{\prime}, y^{\prime}, z^{\prime}, y^{*}, z^{*}\right)$ satisfying all the constraints of (MWD). Let $W_{1}:=\left\{y^{\prime} \in F\left(x^{\prime}\right):\left(x^{\prime}, y^{\prime}, z^{\prime}, y^{*}, z^{*}\right) \in K_{1}\right\}$.

Definition 4.1.1 A feasible point $\left(x^{\prime}, y^{\prime}, z^{\prime}, y^{*}, z^{*}\right)$ of the problem (MWD) is said to be a weak maximizer of (MWD) if

$$
\left(W_{1}-y^{\prime}\right) \cap \operatorname{int}(D)=\emptyset .
$$

We begin with presenting the following weak duality theorem between (MWD) and (SOP).

Theorem 4.1.1 (Weak Duality) Let $\left(x^{\prime}, y^{\prime}\right) \in \operatorname{graph}(F)$ and $z^{\prime} \in G\left(x^{\prime}\right) \cap-E$. Suppose that $(\bar{x}, \bar{y})$ is a feasible solution of (SOP) and $\left(x^{\prime}, y^{\prime}, z^{\prime}, y^{*}, z^{*}\right)$ is a feasible solution of (MWD). Then

$$
\bar{y}-y^{\prime} \notin-\operatorname{int}(D) .
$$

Proof We prove it by contradiction. Suppose

$$
\bar{y}-y^{\prime} \in-\operatorname{int}(D) .
$$

We derive from $y^{*} \in D^{*} \backslash\{0\}$ that

$$
y^{* T}\left(\bar{y}-y^{\prime}\right)<0 .
$$

For the feasible solution $(\bar{x}, \bar{y})$ of (SOP), we get from Lemma 2.1 that

$$
\left((F \times G)(\bar{x})-\left(y^{\prime}, z^{\prime}\right)\right) \subset D_{R}^{m}(F \times G)\left(x^{\prime}, y^{\prime}, z^{\prime}\right)\left(\bar{x}-x^{\prime}\right) .
$$

Since $G(\bar{x}) \cap-E \neq \emptyset$, taking $\bar{z} \in G(\bar{x}) \cap-E$, we obtain from the constraint $z^{*} \in E^{*}$ that

$$
z^{* T} \bar{z} \leq 0 .
$$

Therefore, due to the constraint $z^{* T} z^{\prime} \geq 0$ (the second constraint condition in (MWD)), we have that

$$
z^{* T}\left(\bar{z}-z^{\prime}\right) \leq 0 .
$$

Furthermore, it yields from the first constraint of (MWD) that

$$
y^{* T}\left(\bar{y}-y^{\prime}\right)+z^{* T}\left(\bar{z}-z^{\prime}\right) \geq 0 .
$$

So, we get

$$
y^{* T}\left(\bar{y}-y^{\prime}\right) \geq 0 .
$$

This is a contradiction.

Theorem 4.1.2 (Strong duality) Let $(\bar{x}, \bar{y}) \in \operatorname{graph}(F)$ and $\bar{z} \in G(\bar{x}) \cap(-E)$ and $(F-\bar{y}) \times G$ be near $D \times E$ subconvexlike on $S$. Suppose that $(\bar{x}, \bar{y})$ is a weak minimizer of (SOP) and $\operatorname{cl}(\operatorname{cone}(G(S)+E))=Z$. Then there exists $y^{*} \in D^{*} \backslash\{0\}$ and $z^{*} \in E^{*}$ such that $\left(\bar{x}, \bar{y}, \bar{z}, y^{*}, z^{*}\right)$ is a feasible solution of (MWD). Furthermore, if the Weak Duality Theorem 4.1.1 holds, then $\left(\bar{x}, \bar{y}, \bar{z}, y^{*}, z^{*}\right)$ is a weak maximizer of (MWD).

Proof Firstly, by Theorem 3.2, there are $y^{*} \in D^{*} \backslash\{0\}$ and $z^{*} \in E^{*}$ such that $\left(\bar{x}, \bar{y}, \bar{z}, y^{*}, z^{*}\right)$ is a feasible solution of (MWD). We only need to prove that $\left(\bar{x}, \bar{y}, \bar{z}, y^{*}, z^{*}\right)$ is a weak maximizer of (MWD). We prove this by contradiction. If there exists a feasible solution $\left(x_{0}, y_{0}, z_{0}, y_{0}^{*}, z_{0}^{*}\right)$ of (MWD) such that

$$
y_{0}-\bar{y} \in \operatorname{int} D \text {, }
$$

then

$$
\bar{y}-y_{0} \in-\operatorname{int} D,
$$

which contradicts the Weak Duality Theorem 4.1.1.

Theorem 4.1.3 (Converse duality) Let $\left(x^{\prime}, y^{\prime}\right) \in \operatorname{graph}(F)$ and $z^{\prime} \in G\left(x^{\prime}\right) \cap(-E)$. If there exists $y^{*} \in D^{*} \backslash\{0\}$ and $z^{*} \in E^{*}$ such that $\left(x^{\prime}, y^{\prime}, z^{\prime}, y^{*}, z^{*}\right)$ is a feasible solution of (MWD), then $\left(x^{\prime}, y^{\prime}\right)$ is a weak minimizer of (SOP).

Proof It can be proved directly by utilizing Theorem 3.3. 


\subsection{Wolfe Type Duality}

Let us fix a point $d_{0} \in D \backslash\{0\}$. Suppose that $\left(x^{\prime}, y^{\prime}\right) \in \operatorname{graph}(F)$ and $z^{\prime} \in G\left(x^{\prime}\right) \cap-E$. Considering the following problem (WD), which is called Wolfe type dual problem of (SOP):

$$
\text { (WD) }\left\{\begin{array}{cc}
\max & y^{\prime}+z^{* T} z^{\prime} \cdot d_{0} \\
\text { s.t. } & y^{* T} y+z^{* T} z \geq 0, \forall(y, z) \in D_{R}^{m}(F \times G)\left(x^{\prime}, y^{\prime}, z^{\prime}\right)(x), \\
& \forall x \in \operatorname{dom} D_{R}^{m}(F \times G)\left(x^{\prime}, y^{\prime}, z^{\prime}\right) \\
& y^{* T} d_{0}=1, \\
& \left(y^{*}, z^{*}\right) \in\left(D^{*} \backslash\left\{0_{Y^{*}}\right\}\right) \times E^{*} .
\end{array}\right.
$$

Denote by $K_{2}$ the set of all feasible points of (WD), i.e. the set of points $\left(x^{\prime}, y^{\prime}, z^{\prime}, y^{*}, z^{*}\right)$ satisfying all the constraints of Problem (WD). Let $W_{2}=\left\{y^{\prime}+z^{* T} z^{\prime} \cdot d_{0}:\left(x^{\prime}, y^{\prime}, z^{\prime}, y^{*}, z^{*}\right) \in K_{2}\right\}$.

Definition 4.2.1 A feasible point $\left(x^{\prime}, y^{\prime}, z^{\prime}, y^{*}, z^{*}\right)$ of the problem (WD) is said to be a weak maximizer of (WD) if

$$
\left(W_{2}-\left(y^{\prime}+z^{* T} z^{\prime} \cdot d_{0}\right)\right) \cap \operatorname{int}(D)=\emptyset .
$$

Theorem 4.2.1 (Weak Duality) Let $\left(x^{\prime}, y^{\prime}\right) \in \operatorname{graph}(F)$ and $z^{\prime} \in G\left(x^{\prime}\right) \cap-E$. Suppose that $(\bar{x}, \bar{y})$ and $\left(x^{\prime}, y^{\prime}, z^{\prime}, y^{*}, z^{*}\right)$ be feasible points for (SOP) and (WD), respectively. Then

$$
\bar{y}-y^{\prime}-z^{* T} z^{\prime} \cdot d_{0} \notin-\operatorname{int} D .
$$

Proof Firstly, since $\bar{x} \in S$ and $G(\bar{x}) \cap(-E) \neq \emptyset$, we get from Lemma 2.1 that

$$
\left((F \times G)(\bar{x})-\left(y^{\prime}, z^{\prime}\right)\right) \subset D_{R}^{m}(F \times G)\left(x^{\prime}, y^{\prime}, z^{\prime}\right)\left(\bar{x}-x^{\prime}\right) .
$$

Taking $\bar{z} \in G(\bar{x}) \cap(-E)$, it follows from the first constraint of problem (WD) that

$$
y^{* T}\left(\bar{y}-y^{\prime}\right)+z^{* T}\left(\bar{z}-z^{\prime}\right) \geq 0 .
$$

Suppose

$$
\bar{y}-\left(y^{\prime}+z^{* T} z^{\prime} \cdot d_{0}\right) \in-\operatorname{int} D .
$$

Because $z^{* T} \bar{z} \leq 0$, we get that $z^{* T} \bar{z} \cdot d_{0} \in-D$ and

$$
\bar{y}+z^{* T} \bar{z} \cdot d_{0}-\left(y^{\prime}+z^{* T} z^{\prime} \cdot d_{0}\right) \in-D-\operatorname{int} D \subset-\operatorname{int} D .
$$

Noticing that $y^{*} \in D^{*} \backslash\left\{0_{Y^{*}}\right\}$ and $y^{* T} d_{0}=1$, we have

$$
y^{* T}\left(\bar{y}-y^{\prime}\right)+z^{* T}\left(\bar{z}-z^{\prime}\right)<0,
$$

which is a contradiction. Thus, we obtain $\bar{y}-y^{\prime}-z^{* T} z^{\prime} \cdot d_{0} \notin-\operatorname{int}(D)$.

Theorem 4.2.2 (Strong duality) Let $(\bar{x}, \bar{y}) \in \operatorname{graph}(F)$ and $\bar{z} \in G(\bar{x}) \cap(-E)$. Suppose that $(\bar{x}, \bar{y})$ is a weak minimizer of (SOP) and that for some $\left(y^{*}, z^{*}\right) \in\left(D^{*} \backslash\{0\}\right) \times E^{*}$ with $y^{* T} d_{0}=1$ such that for all $(y, z) \in$ $D_{R}^{m}(F \times G)(\bar{x}, \bar{y}, \bar{z})(x), x \in \operatorname{dom} D_{R}^{m}(F \times G)(\bar{x}, \bar{y}, \bar{z})$,

$$
y^{* T} y+z^{* T} z \geq 0,
$$

and

$$
z^{* T} \bar{z}=0 .
$$

Then, $\left(\bar{x}, \bar{y}, \bar{z}, y^{*}, z^{*}\right)$ is a feasible solution for (WD). Furthermore, if the Weak Duality Theorem 4.2.1 holds, then $\left(\bar{x}, \bar{y}, \bar{z}, y^{*}, z^{*}\right)$ is a weak maximizer of (WD). 
Proof It is obviously that $\left(\bar{x}, \bar{y}, \bar{z}, y^{*}, z^{*}\right)$ is a feasible solution of (WD) and

$$
z^{* T} \bar{z}=0 .
$$

Next, we show that

$$
\left(W_{2}-\bar{y}-z^{* T} \bar{z} \cdot d_{0}\right) \cap \operatorname{int} D=\emptyset .
$$

Let $\left(x^{\prime}, y^{\prime}, z^{\prime}, y_{1}^{*}, z_{1}^{*}\right)$ be a feasible solution for (WD) such that

$$
y^{\prime}+z_{1}^{* T} z^{\prime} \cdot d_{0} \in\left(W_{2}-\bar{y}-z^{* T} \bar{z} \cdot d_{0}\right) \cap \operatorname{int} D .
$$

It yields from $z^{* T} \bar{z}=0$ that

$$
y^{\prime}+z_{1}^{* T} z^{\prime} \cdot d_{0} \in\left(W_{2}-\bar{y}\right) \cap \operatorname{int}(D) .
$$

Therefore,

$$
y^{\prime}+z_{1}^{* T} z^{\prime} \cdot d_{0}-\bar{y} \in \operatorname{int}(D) .
$$

This contradicts the Weak Duality Theorem 4.2.1.

Theorem 4.2.3 (Converse duality) Let $\left(x^{\prime}, y^{\prime}\right) \in \operatorname{graph}(F)$ and $z^{\prime} \in G\left(x^{\prime}\right) \cap(-E)$. If there exists $y^{*} \in D^{*} \backslash\{0\}$ and $z^{*} \in E^{*}$ such that $\left(x^{\prime}, y^{\prime}, z^{\prime}, y^{*}, z^{*}\right)$ is a feasible solution of (MWD) and $z^{* T} z^{\prime}=0$, then $\left(x^{\prime}, y^{\prime}\right)$ is a weak minimizer of (SOP).

Proof It can be proved directly by using Theorem 3.3.

\section{Acknowledgement}

This research was supported by Natural Science Foundation of China under Grant (No. 11361001); Natural Science Foundation of Ningxia under Grant (No. NZ14101).

\section{REFERENCES}

1. S. J. Li, K. L. Teo, X. Q. Yang, Higher-order optimality conditions for set-valued optimization, Journal of Optimization Theory and Applications, 137: 533-553, 2008.

2. Q. L. Wang, S. J. Li, K. L. Teo, Higher-order optimality conditions for weakly efficient solutions in nonconvex set-valued optimization, Optimization Letters, 4: 425-437, 2010.

3. S. J. Li, K. L. Teo, X. Q. Yang, Higher-order Mond-Weir duality for set-valued optimization, Journal of Computational and Applied Mathematics, 217: 339-349, 2008.

4. S. J. Li, C. R. Chen, Higher order optimality conditions for Henig efficient solutions in set-valued optimization, Mathematical Analysis and Applications, 323: 1184-1200, 2006.

5. C. R. Chen, S. J. Li, K. L. Teo, Higher order weak epiderivatives and applications to duality and optimality conditions, Computers and Mathematics with Applications, 57: 1389-1399, 2009.

6. P. Q. Khanh, N. D. Tuan, Variational sets of multivalued mappings and a unified study of optimality conditions, Journal of Optimization Theory and Applications, 139: 45-67, 2008.

7. P. Q. Khanh, N. D. Tuan, Higher-order variational sets and higher-order optimality conditions for proper efficiency in set-valued nonsmooth vector optimization, Journal of Optimization Theory and Applications, 139: 243-261, 2008.

8. Nguyen Le Hoang Anh, Phan Quoc Khanh, Le Thanh Tung, Variational sets: Calculus and applications to nonsmooth vector optimization, Nonlinear Analysis, 74: 2358-279, 2011.

9. D. V. Luu, Higher-order necessary and sufficient conditions for strict local Pareto minima in terms of Studniarskis derivatives, Optimization, 57: 593-605, 2008

10. X. K. Sun, S. J. Li, Lower Studniarski derivative of the perturbation map in parametrized vector optimization, Optimization Letters, 5: 601-614, 2011.

11. Nguyen Le Hoang Anh, Higher-order optimality conditions in set-valued optimization using Studniarski derivatives and applications to duality, Positivity, 18: 449-473, 2014.

12. M. Studniarski, Necessary and sufficient conditions for isolated local minima of nonsmooth functions, SIAM J. Control Optim., 24: 1044-1049, 1986. 
13. Guolin Yu, Higher-order optimality conditions and duality for approximate solutions in non-convex set-valued optimization, Acta Mathematicae Applicatae Sinica, to appear.

14. A. Taa, Set-valued derivatives of multifunctions and optimality conditions, Numerical Functional Analysis and Optimization, 19: 121-140, 1998.

15. F. Flores-Bazan, Radial epiderivatives and asymptotic function in nonconvex vector optimization, SIAM J. Optim. 14: 284-305, 2003.

16. R. Kasimbeyli, Radial epiderivatives and set-valued optimization, Optimization, 58: 521-534, 2009

17. F. Flores-Bazan, B. Jimenez, Strict efficiency in set-valued optimization, SIAM J. Control Optim. 48: 881-908, 2009

18. Nguyen Le Hoang Anh, Phan Quoc Khanh, Le Thanh Tung, Higher-order radial derivatives and optimality conditions in nonsmooth vector optimization, Nonlinear Analysis, 74: 7365-7379, 2011.

19. Nguyen Le Hoang Anh, Phan Quoc Khanh, Higher-order optimality conditions in set-valued optimization using radial sets and radial derivatives, Journal of Global Optimization, 56: 519-536, 2013.

20. Nguyen Le Hoang Anh, Phan Quoc Khanh, Higher-order optimality conditions for proper efficiency in nonsmooth vector optimization using radial sets and radial derivatives, Journal of Global Optimization, 58: 693-709, 2014.

21. H. W. Corley, Existence and Lagrangian duality for maximization of set-valued functions, Journal of Optimization Theory and Applications, 54: 489-501, 1987.

22. D. Bhatia, A. Mehra, Lagrangian duality for preinvex set-valued functions, Journal of Mathematical Analysis and Applications, 214: 599-612, 1997.

23. Z. F. Li, G. Y. Chen, Lagrangian multipliers, saddle points, and duality in vector optimization of set-valued maps, Journal of Mathematical Analysis and Applications, 215: 297-316, 1997.

24. Z. F. Li, Benson proper efficiency in the vector optimization of set-valued maps, Journal of Optimization Theory and Applications, 98: 623-649, 1998.

25. X. M. Yang, D. Li, S. Y. Wang, Near-subconvexlikeness in vector optimization with set-Valued functions, Journal of Optimization Theory and Applications, 110: 413-427, 2001.

26. María Alonso-Durán, Luis Rodríguez-Marín, On approximate solutions in set-valued optimization problems, Journal of Computational and Applied Mathematics, 2012, 236: 4421-4427.

27. P. H. Sach, New generalized convexity notion for set-valued maps and application to vector optimization, Journal of Optimization Theory and Applications, 125: 157-179, 2005.

28. Guolin Yu, Sanyang Liu, Globally proper saddle point in ic-cone-convex like set-valued optimization problems, Acta Mathematica Sinica, English Series, 25: 1921-1928, 2009. 